\title{
New category of evidence should permit the multinational association of support in cancer care (MASCC) to review polymerized cross-linked sucralfate paste (ProThelial ${ }^{\mathrm{m}}$ ) for mucositis guidelines
}

Ricky W. McCullough

Correspondence: glencopelph@aol.com

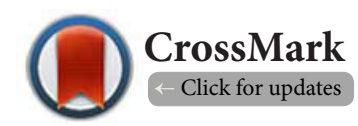

Center for Translational Medicine Research, Mueller Medical International LLC, United States.

Department of Medicine and Emergency Medicine, Veterans Administration Hospital, Brown University Teaching Hospital, Providence Rhode Island.

\begin{abstract}
Background: Mucositis is a significant burden for cancer treatment patients. Due to suboptimal interventions mucositis remains an insurmountable challenge for oncologists forcing dose reductions, treatment postponement or cancellation all of which directly impact clinical outcomes and survival.

New mucositis intervention with no clinical guideline status: Polymerized cross-linked sucralfate (PCLS) paste recently cleared by the US FDA for the management of oral mucositis. Mucositis registry data has been repeatedly associated PCLS with rapid reversal and complete prevention of oral mucositis. However the level evidence used are observational data from a mucositis registry. Evidence from randomized clinical trials is the goal standard of efficacy for inventions, except those interventions wherein the magnitude of clinical effect are dramatic, that is beyond 10 times that expected by placebo. In such circumstances efficacy of such interventions can be statistical assured with from non-randomized observational study, not level evidence commonly ranked high.

Discussion: Observational data on interventions with dramatic clinical effects (10 times that of placebo) should be included as a superior level of evidence appropriate for guideline recommendations of the Multinational Association of Support in Cancer Care/International Society of Oral Oncology (MASCC/ ISOO). This call for revision in the categories of evidence by the MASCC/ISOO could compel discussion and possible recommendation for the use of PCLS in the management of oral mucositis. In turn, such a recommendation may possibly impact cancer treatment outcomes in patients with mucositis.

Conclusion: This article probes the pros and cons of this point decidedly in favor of establishing a Level IA evidence category for mucositis interventions. Repeated demonstrations of rapid, complete and simultaneous reversal of oral and alimentary mucositis within two to three days in 25 cancer treatment patient has prompted this suggestion.
\end{abstract}

Keywords: Sucralfate, mucositis, MASCC/ISOO, glasziou rate ratio, mucositis clinical guidelines

\section{Introduction}

The Mucositis Panel of the Multi-national Association for Supportive Cancer Care and the International Society Oral Oncology Cancer (MASCC/ISOO) publishes and periodically updates practice guidelines for practitioners regard the management of mucositis in cancer treatment patients. In 2013 the MASCC/ ISOO Panel [1] discussed their efforts in the systemic reviews on mucositis interventions and the evidence criterion used to make practice recommendation or suggestions. For interventions with clinical effects that are several folds greater in magnitude 
Ricky W. McCullough, Oncology Discovery 2014,

than placebo, randomized clinical trials are considered as level 1 evidence. Any observational trials on the same quality of interventions are rated as level 5, the lowest level of evidence. Overlooked as a higher level of evidence are non-randomized observational trials on interventions with clinical effects that are at least 1000 times better than placebo. The point of this debate is that, for such interventions, non-randomized observational trials are sufficient to establish efficacy and should be sufficient to justify discussion by the Panel for inclusion in the guidelines. Indeed rare are such interventions, however a recently FDA cleared intervention ProThelial or polymerized cross-linked sucralfate (PCLS) medical device may qualify as such an intervention.

In five patients, PCLS was consistently associated with 2-3 day reversal of mucositis instead of the typical 50-70 days required for resolution if left to its natural course. This reduction in the course of mucositis establishes efficacy according to the Glasziou treatment effect [2], which supports as adequate evidence the use of non-randomized observational trials for this type of intervention.

\section{Current MASCC criteria used to evaluate literature evidence}

The current criterion used by the MASCC Panel has been published [1]. The Panel used suggestions from Hadorn et al., [3] to direct its analysis of published research and adopted the criteria outlined by Somerfield et al., [4] to assign levels of evidence on published investigations. Table 1 outlines the criterion used to assign levels to the evidence gathered from published articles.

The vast majority of interventions studied by randomized clinical trial (RCT) give rise to treatment effects that are only 20-50 base points better than placebo that is between oneto two-fold better than placebo. That specific magnitude of treatment effect, that is, $200 \%-300 \%$ beyond placebo, can be overpowered by biases of methodology or trial design. Historically there rare interventions are ever associated with a magnitude of treatment effects that are beyond, 20-50 base points, that is, beyond one-to two-fold greater (or 200-300\% better) than placebo. Ergo, there is generalized comfort among investigators that RCT is the unique determinant of clinical efficacy for all interventions. In other words, a well-designed
RCT is absolutely required to assess efficacy in any (and all) all interventions.

The stratification of level of evidence used by the MASCC (in Table 1) makes sense, because the magnitude of clinical effects for the vast majority interventions is "average".

However, unknown to most investigators is that there is a magnitude of clinical effect caused by any intervention that statistically annuls the need of RCT to confirm efficacy. This is a little known fact, but a fact nonetheless.

\section{Discussion}

The position of this report and the heart of the debate is that the Panel (unintentionally) excluded statistically valid evidence of efficacy generated by observational trials on interventions that cause "dramatic" clinical effects. The implication of using Table 1 as the sole determinant of quality of evidence, the MASCC Panel overlooked the rare instance wherein the magnitude of clinical effect from an intervention could be large enough to drown out any confounding influence by bias. In so doing, the Panel has endorsed a widespread erroneous and non-evidence-based view that RCT have infallible discernment of efficacy for all interventions.

For interventions wherein the magnitude of clinical effect is "average", this position is true and evidence-based. For interventions where the magnitude of clinical effect is "above average", that is dramatic, this view is not true and not evidence-based. A measure of the magnitude of clinical effect discussed by Glasziou et al., [2] is known as the rate ratio stated, if the rate in which the clinical effect caused by an intervention is more than or equal to 10 times the clinical effect that occurs with placebo, then that clinical effect is "dramatic" or beyond average. Such an effect regardless as to how it was observed -by RCT or by observational trial -is a statistically significant mark of efficacy, particularly if that effect is repeatedly observed under multiple clinical settings.

However, Table 1 utilized by the Panel, in its current format, does not reflect a true evidence-based standard of assessing clinical efficacy.

\section{Common error of non-evidence based use of randomized clinical trials (RCT) \\ There is a common error of non-evidence base insistence}

Table 1. Levels Of Evidence Used by MASCC/ISOO Panel on Mucositis Guidelines.

\footnotetext{
I Evidence obtained from meta-analysis of multiple, well-designed, controlled studies; randomized trials with low false-positive and false-negative errors (high power)

II Evidence obtained from at least one well-designed experimental study; randomized trials with high falsepositive and /or false-negative errors (low power)

III Evidence obtained from well-designed, quasi-experimental studies, such as nonrandomized, controlled single-group, pretest-posttest comparison, cohort, time or matched case-control series

IV Evidence obtained from well-designed, non-experimental studies, such as comparative and correlational descriptive and case studies

V Evidence obtained from case reports and clinical examples
} 
of RCTs for interventions whose magnitude of clinical effect far exceeds confounding background noise of placebo, and biases of design and non-randomization. However, scarcely appreciated is that, for interventions wherein the magnitude of clinical effect is "large" and beyond "average" RCTs are statistically incapable of refuting efficacy verified by obser-vational trials on such interventions. "Average" is defined as clinical magnitudes 1000 times of placebo [4]. In the case above "average" interventions, those associated with a larger than average magnitude of clinical effect, the insistence of RCT to identify efficacy has no rational support in evidence-based medicine.

Randomized clinical trials to confirm efficacy of parachutes Smith and Pell [5] sought to illustrate that the insisted use of RCT is limited by the magnitude of clinical effect observed in any one case report. The inevitability of sure bodily harm or death is convincingly proven by the single use of a parachute, the magnitude of the clinical effect being stark, similarly to the use of antibiotics for infections, and the use of insulin for juvenile diabetics. Smith and Pell artfully demonstrated that the magnitude of clinical effect observed in any one single case report (that is the prevention of death or major trauma by parachute) precludes any requirement on insistence of RCT as proof of efficacy of parachutes. Using as data sources Medline, Web of Science and Cochrane Library databases, and a main outcome measure of death or major trauma defined by an injury severity score of 15 , they were "unable to identify any randomized controlled trials of parachute intervention". Declaring that the effectiveness of parachutes had not been subjected to rigorous evaluation by an RCT, they proposed that 'all would benefit if the most radical protagonists of evidence based medicine organized and participated in a double-blind, randomized, placebo controlled, crossover trial on the parachute'.

Behind the humor of the article is the understated point that the clinical magnitude of the effect by any intervention that excludes it from efficacy-evaluation by an RCT and that this exclusion of otherwise required an RCT is evidence-based medicine.

\section{Hazard ratio in clinical trials}

When the extent of treatment can shorten the duration of the illness, clinicians use Hazard ratios to describe the outcome of therapeutic trials, whether randomized or observational.

Therapeutic outcomes of trials can be discussed either in terms of magnitude of clinical effect of an intervention compared to placebo, or in terms of the shortness of illness duration once an intervention used.

In a mini-review, Spruance et al., [6] discussed that trial outcome of interventions can be compared on the basis of the time of symptom resolution. Instead of the margin of efficacy between interventions, outcomes can be viewed in terms of the odds of symptom resolution of illness at time ' $x$ ' of applying intervention.

Owing to the poor efficacy of most mucositis intervention, once mucositis occurs during the course of cancer treatment, mucositis will persist $60-70$ days $[7,8]$. Neither placebo nor mucositis rinses have altered this course. However, PCLS has been associated with 2-3 day reversal of oral mucositis and radiation oncologists have used PCLS to completely prevention oral mucositis in patients in an open mucositis registry in the US [9].

In a mucositis patient that would customarily require 60-70 days before resolution, if the patient was using s nnn-PCLS intervention (or placebo), then the hazard-based odds of mucositis resolution is very small, perhaps $1 / 60$ or $1 / 70$ or $1.4 \%$ to $1.7 \%$ on day 2 or 3 . Conversely, given the reported experience of PCLS in the national mucositis registry, in an oral mucositis patient using the PCLS intervention, the hazard-based odds of complete disease resolution is just the opposite, that is $1-(1 / 60)$ to $1-(1 / 70)$ or $98.3 \%-98.6 \%$, which is high. This magnitude of clinical effect using PCLS is so great that in terms of the Hazard ratio, one expects that on day 3 of an customary 70 day course, there are $98 \%$ odds of disease resolution. In such a case, it is not likely that biases controlled by RCTs (that is, blinding, randomization of selection) will over come those odds.

Again, the "magnitude of clinical effect of an intervention" is determinant to the relevance of a RCT to confirm efficacy.

\section{The debate's critical point}

The critical point of this debate is this mutually inclusive conclusion. Firstly, there is a statistically relevant boundary where in only RCTs in deference to observational trials can identify efficacy and secondly, there is a statistically equivalent boundary of clinical effect wherein efficacy can be securely identified by observational trials alone. That boundary is determined by the magnitude of the clinical effect of an intervention compared to the magnitude of clinical effect of placebo. This is a little known, but irrefutable, statistical truth that the author seeks to highlight in this article.

The basic position of the author is that, while ineffective in validating efficacy of interventions of "average" clinical effects, observational trials are statistically trustworthy to validated interventions that cause "large clinical effects" compared to placebo. The statistical relevance of these "large clinical effects" have been addressed by the work of Professor Paul Glasziou, director of evidence-based medicine (Oxford University, Oxford UK and Bond University of Australia) [2].

The published criterion for evidence (Table 1) used by the MASCC mucositis Panel does not accommodate this fact, that observation trials on interventions that have large clinical effects should be considered as Level I medical evidence.

\section{Common oversight: the magnitude of clinical effect corroborates efficacy and the innecessity of RCT}

The magnitudes of clinical effect of an intervention corroborates 
Ricky W. McCullough, Oncology Discovery 2014,

efficacy and the innecessity of an RCT to prove or establish efficacy. For modest or average magnitudes of clinical effects, those that are 1-8 fold greater than placebo, then RCTs are required to confirm efficacy. Likewise, there are magnitudes of clinical effect by an intervention that invalidates the need of an RCT to confirm efficacy.

Most are unaware (as the author had been) that when the magnitude of clinical effect of any intervention is less than $800 \%$, then an RCT is required to verify efficacy. Equally, most are unaware that when the magnitude of clinical effect of an intervention supersedes $1000 \%$ the effect of placebo, then performance of RCT will only confirm efficacy proven by multiple observation trials. In other words, a RCT in such a circumstance will not add to confidence regarding efficacy confirmed already by observational data.

The use of RCT to assess efficacy operates under the presumption that the magnitude of clinical effect of any intervention is far less that $1000 \%$ the treatment effect of placebo.

So what happens if the magnitude of clinical effect of an intervention is $1000 \%$ beyond that of placebo? Then an RCT is useful for repetition, for regulatory approval, for the study of adverse reactions incidental to the use of the intervention, but it is not statistically necessary to confirm or prove efficacy. In other words, it is against the principle of evidence-based medicine to insist on RCT proof of efficacy for an intervention if the magnitude of clinical effect of that intervention is $1000 \%$ beyond that of placebo.

When is the insistence of an RCT for an intervention purely emotional and opposes the tenets of evidence-based medicine? Some proponents of RCTs contend never and such proponents in one particular circumstance would be found to be are emotional pragmatics against evidence-based medicine.

Are there indeed, any circumstances wherein insistence of an RCT for an intervention is against evidence-based medicine? Paul Glasziou, professor of evidence-based medicine, asserts that there is. He is not alone. Several have discussed this point wherein the use of an RCT to prove efficacy demonstrated by an observational trial was not necessary. In each instance the turning point was the "magnitude of the clinical effect" observed.

Application: observational data form the use of poly merized cross-linked sucralfate (PCLS or ProThelial ${ }^{\mathrm{TM}}$ ) High-potency PCLS is standard sucralfate polymerized into 'sucralfate sheets' by bidentate malate chelator (sequestering sucralfate from aqueous solution) and then cross-linked by chelated calcium resulting in a preferentially ordered layering of sucralfate on the mucosa. PCLS achieves and maintains elevated surface concentrations of sucralfate long after the initial dose. Three hours following administration, PCLS maintains a seven-fold (or $800 \%$ ) greater surface concentration of sucralfate on normal lining and a 23 -fold (or 2,400\%) greater concentration on inflamed, ulcerated mucosa [10].

Standard potency non-polymerized sucralfate is not recom- mended by MASCC/ISOO for the treatment or prevention of mucositis, oral or gastrointestinal. The author supports this position [11]. However, high potency polymerized cross-linked sucralfate has a 2-3 day time of resolution compared to 60-70 days for placebo. It is not the same intervention as standard potency non-polymerized sucralfate as it is associated with rapid reversal of both oral and alimentary mucositis in patients.

PCLS (ProThelial ${ }^{\mathrm{TM}}$ ) is FDA cleared as a medical device to be used orally and then expectorated, though it is perfectly safe to swallow. Contrary to the package insert medical oncologists have instructed patients to swish and swallow PCLS, and have reported associated resolution of both oral and gastrointestinal mucositis. Radiation oncologists have reported sustained prevention of oral mucositis while under active radiotherapy.

As required by regulation, the prescription instructions assert the safety of swallowing ProThelial ${ }^{\mathrm{TM}}$, though not promoting it. The FDA-cleared quantity of sucralfate per dose of PCLS is $250 \mathrm{mg}$ to $500 \mathrm{mg}$ used three times daily for the first day, followed by the same amount used twice daily. This is in contrast to customary FDA-approved ingested dosing of standard-potency sucralfate of $1,000 \mathrm{mg}$ swallowed four times daily.

Observational trials were used to assess the clinical efficacy of PCLS in Phase IV post-market surveillance in a Mucositis Registry. This Registry revealed greatly shortened times of symptom resolution using ProThelial ${ }^{\mathrm{TM}}$. As mentioned earlier, once established during continued cancer treatment, oral mucositis persists for $60-70$ days $[7,8]$. The use of PCLS (ProThelial ${ }^{\mathrm{TM}}$ ) in 5 out of 5 reporting cases [9] the use of PCLS was associated with the reversal of oral mucositis in two to three days or was associated with complete prevention of mucositis during cancer treatment. Table 2 shows a five patient excerpt of a total of 5 patients using PCLS in the national mucositis registry..

These case excerpts were selected to demonstrate the extreme case differences in which the same clinical outcomes were obtained using PCLS. It would appear that 2-3 day alleviation of mucositis usually requiring 60-70 days to do so, qualifies PCLS as an intervention capable of repeated an above "average" treatment outcomes.

MASCC panel exclusion of positive glasziou treatment effects in observational trials is not evidence-based medicine

The categorization of evidence outlined in Table 1 is complete for interventions whose treatment effects that are tens of percentage-point better than placebo.

Table 1 excludes evidence-based outcomes arising from case reports exhibiting large magnitude of clinical effects, which are treatment effects that statistically outstrip the influence of bias of trial design or random chance.

\section{Other reasons to support MASCC discussions to recom- mend use of PCLS}

There are several other reasons supporting the inclusion of 
observational trial data Panels discussion of the use of PCLS in patients with oral and alimentary mucositis.

First, PCLS is fast, affecting complete reversal of signs and symptoms of mucositis within two to three days. Second, PCLS appears useful simultaneously for both OM and GIM. Third, the treatment effect of PCLS appears wider in scope than any other mucositis intervention to date. It eliminates pain, restores normal oral mucosa, and restores upper GI function with swallowing and the ability to tolerate solids and liquids. This feature alone permits the patients to self-maintain their nutritional status while undergoing cancer treatment. Additionally, in these patients PCLS minimized nausea and small intestinal cramping and it eliminated frequent loose movements in patients suffering from chemotherapy-induced diarrhea. Fourth, in these patients, PCLS seems useful for the management of mucositis caused by a diverse group of oncologic agents each having differing mechanisms of action. Cancer therapy included radiation, 5-fluorouracil, folinic acid, irinotecan, oxaliplatin, paclitaxel, carboplatin, cetuximab, ipilimumab and nivolumab. Fifth, PCLS was well-tolerated by all, with no patient-reported side effects. Of course, sucralfate, the active ingredient of PCLS, has an acceptable safety profile.
Sixth, PCLS is a singular agent with an acceptable means of administration, dosed at a frequency that is not cumbersome.

\section{Suggested alternative to be used by MASCC as level of evidence}

Table 3 is a suggested new level of evidence to be considered by the MASCC/ISOO Panel for mucositis guidelines. historically, interventions that generate treatment effects that exemplify IA level evidence include insulin for diabetics, neostigmine for myasthenia gravis or penicillin for infections. The latter involved the study of 10 patients, wherein five were given penicillin intravenously, one orally and four topically [12] More recent case-report data that alter guidelines of clinical practice include the use of 2-chlorodeoxyadenosine for remission of hairy-cell leukemia [13], the use of imatinib in the treatment of chronic myeloid leukemia [14] and the use of combination chemotherapy to treat advanced Hodgkin's disease [15].

Indeed, there are case reports that demonstrate rate ratios beyond the power of 10 and the evidence criterion used to base clinical guidelines should accommodate such occurrences that occur in medical research. Provided known or demonstrated

Table 2. Patients on ProThelial Polymerized Cross Linked Sucralfate Status-Post Market Clearance.

\begin{tabular}{|c|c|c|c|c|c|c|c|c|c|c|}
\hline Patient & $\begin{array}{l}\text { Age } \\
\text { Gender }\end{array}$ & $\begin{array}{l}\text { Institution } \\
\text { University }\end{array}$ & Cancer & $\begin{array}{l}\text { Oncology } \\
\text { Treatment }\end{array}$ & $\begin{array}{l}\text { Oral } \\
\text { Mucositis }\end{array}$ & $\begin{array}{l}\text { Gastrointestinal } \\
\text { Mucositis }\end{array}$ & $\begin{array}{l}\text { Reversal of } \\
\text { Ulceration }\end{array}$ & $\begin{array}{l}\text { Reversal } \\
\text { of Painful } \\
\text { Swallowing }\end{array}$ & $\begin{array}{l}\text { Reversal } \\
\text { of Nausea, } \\
\text { Cramps } \\
\end{array}$ & $\begin{array}{l}\text { Reversal } \\
\text { of } \\
\text { Diarrhea }\end{array}$ \\
\hline 1 & 43yoм & $\begin{array}{l}\text { Brown } \\
\text { University } \\
\text { Boston } \\
\text { University }\end{array}$ & SCCHN & $\begin{array}{l}\text { Radiation + } \\
\text { Carboplatin } \\
+ \text { Paclitaxel }\end{array}$ & Grade 2 & Grade 2 & 2 days & 2 days & 1 day & 1 day \\
\hline 2 & 49yoМ & Midwestern & SCCHN & $\begin{array}{l}\text { Radiation+ } \\
\text { Cetuximab }\end{array}$ & Grade 3 & Grade 2 & 2 days & 3 days & 1 day & 1 day \\
\hline 3 & 48yoM & $\begin{array}{l}\text { Vanderbilt } \\
\text { Ingram } \\
\text { CaCtr }\end{array}$ & $\begin{array}{l}\text { Pancreatic } \\
\text { Carcinoma }\end{array}$ & Folfirinox & Grade 4 & Grade 3 & 3 days & 3 days & 2 days & 2 days \\
\hline 4 & 48 yoF & UCo Yale & $\begin{array}{l}\text { Metastatic } \\
\text { Melanoma }\end{array}$ & $\begin{array}{l}\text { Ipilimumab } \\
\text { Nivolumab }\end{array}$ & Grade 3 & Grade 4 & 2 days & 2 days & 2 days & 2 days \\
\hline 5 & 63 yoF & $\begin{array}{l}\text { Vanderbilt } \\
\text { Ingram } \\
\text { CaCtr }\end{array}$ & $\begin{array}{l}\text { Metastatic } \\
\text { Colon Ca }\end{array}$ & Folfox & Grade 3 & Grade 4 & 2 days & 1 day & 2 days & 2 days \\
\hline
\end{tabular}

Table 3. Suggested New Levels Of Evidence for MASCC/ISOO Panel on Mucositis Guidelines.

\begin{tabular}{ll}
\hline IA & Evidence obtained from reports demonstrating rate ratio equal to or more than 10 \\
I B & $\begin{array}{l}\text { Evidence obtained from meta-analysis of multiple, well-designed, controlled studies; randomized trials } \\
\text { with low false-positive and false-negative errors (high power) }\end{array}$ \\
II & $\begin{array}{l}\text { Evidence obtained from at least one well-designed experimental study; randomized trials with high false- } \\
\text { positive and/or false-negative errors (low power) }\end{array}$ \\
III & $\begin{array}{l}\text { Evidence obtained from well-designed, quasi-experimental studies, such as nonrandomized, controlled } \\
\text { single-group, pretest-posttest comparison, cohort, time or matched case-control series }\end{array}$ \\
IV & $\begin{array}{l}\text { Evidence obtained from well-designed, non-experimental studies, such as comparative and correlational } \\
\text { descriptive and case studies }\end{array}$ \\
V & Evidence obtained from case reports and clinical examples \\
\hline
\end{tabular}


Ricky W. McCullough, Oncology Discovery 2014,

http://www.hoajonline.com/journals/pdf/2052-6199-2-1.pdf

doi: 10.7243/2052-6199-2-1

safety of an intervention, this type of accommodation will provide meaningful interventions to the community of practitioners sooner than otherwise possible.

\section{Conclusion}

Inclusion of the Glasziou treatment effect as a high-level criterion for evidence of efficacy will legitimize discussions by the Working Committee regarding this novel mucositis intervention. To date there has been no FDA-approved or cleared mucositis treatment associated with the simultaneously reversal of both oral and alimentary mucositis and that within two to three days. High potency PCLS has that distinction and its dramatic treatment effect is a stark difference from standard potency sucralfate that is not recommended by the Committee on mucositis guidelines. Perhaps it is appropriate for the Committee to now seize upon the observation of a positive Glasziou treatment effect of PCLS as the basis for discussing its place in the clinical mucositis guidelines.

\section{List of abbreviations}

MASCC: Multinational Association of Support in Cancer Care

ISOO: International Society of Oral Oncology

RCT: Randomized clinical trial

FDA: Food and Drug Administration

PCLS: Polymerized cross-linked sucralfate

\section{Competing interests}

The author declares that he has no competing interests.

\section{Acknowledgement}

The author would like to thank Theodore Glanzer for assistance in preparation of manuscript.

Publication history

Editor: Hemant Kumar Bid, The Research Institute Nationwide Children's Hospital, USA.

EIC: Paul J. Higgins, Albany Medical College, USA.

G. J. Peters, VU University Medical Center, Netherlands.

William Chi-shing Cho, Queen Elizabeth Hospital, Hong Kong.

Received: 03-Apr-2014 Final Revised: 22-Jul-2014

Accepted: 25-Jul-2014 Published: 30-Jul-2014

\section{References}

1. Bowen JM, Elad S, Hutchins RD and Lalla RV. Methodology for the MASCC/ISOO Mucositis Clinical Practice Guidelines Update. Support Care Cancer. 2013; 21:303-8. | Article | PubMed

2. Glasziou P, Chalmers I, Rawlins M and McCullough P. When are randomized trials unnecessary? Picking signal from noise. BMJ. 2007; 334:349-351. | Article

3. Hadorn DC, Baker D, Hodges JS and Hicks N. Rating the quality of evidence for clinical practice guidelines. J Clin Epidemiol. 1996; 49:74954. | Article | PubMed

4. Sommerfield M, Padberg J, Pfiser D, Bennett C, Recht A, Smith T, Weeks J, Winn R and Durant J. ASCO clinical practice guidelines: process, progress, pitfalls, and prospects. Classic Papers and Current Comments. 2000; 4:881-886.

5. Smith GC and Pell JP. Parachute use to prevent death and major trauma related to gravitational challenge: systematic review of randomised controlled trials. BMJ. 2003; 327:1459-61. | Article | PubMed Abstract | PubMed Full Text

6. Spruance SL, Reid JE, Grace M and Samore M. Hazard ratio in clinical trials. Antimicrob Agents Chemother. 2004; 48:2787-92. | Article | PubMed Abstract | PubMed Full Text

7. Elting LS, Keefe DM, Sonis ST, Garden AS, Spijkervet FK, Barasch A, Tishler RB, Canty TP, Kudrimoti MK and Vera-Llonch M. Patientreported measurements of oral mucositis in head and neck cancer patients treated with radiotherapy with or without chemotherapy: demonstration of increased frequency, severity, resistance to palliation, and impact on quality of life. Cancer. 2008; 113:2704-13. | Article | PubMed

8. Kushner JA, Lawrence HP, Shoval I, Kiss TL, Devins GM, Lee L and Tenenbaum HC. Development and validation of a Patient-Reported Oral Mucositis Symptom (PROMS) scale. J Can Dent Assoc. 2008; 74:59. | Article I PubMed

9. McCullough RW. Impact of Polymerized Cross-Linked Sucralfate for the Management of Chemo-Radiation Mucositis. Outcome Review of 32 Patient Mucosits Registry. Phase IV Post-market Surveillance of ProThelial. Oncologist. (submitted for review June 2014).

10. Kashimura K and Ozawa K. Sucralfate Preparations. US Patent 5,968,906. Oct 19, 1999.

11. Rubenstein EB, Peterson DE, Schubert M, Keefe D, McGuire D, Epstein J, Elting LS, Fox PC, Cooksley C and Sonis ST. Clinical practice guidelines for the prevention and treatment of cancer therapy-induced oral and gastrointestinal mucositis. Cancer. 2004; 100:2026-46. | Article | PubMed

12. Abraham EP, Chain E, Fletcher CM, Gardner AD, Heatley NG and Jennings MA. Further observation on penicillin. Lancet. 1941; 2:177-88. | Article

13. Piro LD, Carrera CJ, Carson DA and Beutler E. Lasting remissions in hairycell leukemia induced by a single infusion of 2-chlorodeoxyadenosine. $N$ Engl J Med. 1990; 322:1117-21. | Article | PubMed

14. Kantarjian $\mathrm{H}$. Hematologic and cytogenetic responses to imatinib mesylate in chronic myeloid leukemia. N Engl J Med. 2002; 346:545-52. I Article

15. Devita VT, Jr., Serpick AA and Carbone PP. Combination chemotherapy in the treatment of advanced Hodgkin's disease. Ann Intern Med. 1970; 73:881-95. | Article | PubMed

\section{Citation:}

McCullough RW. New category of evidence should permit the multinational association of support in cancer care (MASCC) to review polymerized crosslinked sucralfate paste (ProThelial ${ }^{\mathrm{mm}}$ ) for mucositis guidelines. Oncol Discov. 2014; 2:1.

http://dx.doi.org/10.7243/2052-6199-2-1 